\title{
Prediction of postoperative exacerbation of interstitial pneumonia in patients with lung cancer and interstitial lung disease
}

\author{
AKIRA IYODA $^{1}$, SHI-XU JIANG $^{2}$, HIDEKI AMANO $^{1}$, FUMIHIRO OGAWA $^{1}$, YOSHIO MATUI ${ }^{1}$, \\ NAOMI KUROUZU ${ }^{1}$, HIDENORI HARA ${ }^{1}$, KENJI NEZU ${ }^{1}$, TETSUO MIKAMI ${ }^{2}$, \\ MAKOTO SAEGUSA $^{2}$ and YUKITOSHI SATOH ${ }^{1}$ \\ Departments of ${ }^{1}$ Thoracic Surgery, and ${ }^{2}$ Pathology, Kitasato University, School of Medicine, Kanagawa, Japan
}

Received February 4, 2011; Accepted July 8, 2011

DOI: $10.3892 / \mathrm{etm} .2011 .342$

\begin{abstract}
Postoperative exacerbation of interstitial pneumonia in patients with lung cancer and interstitial lung disease has emerged as a serious problem. Therefore, the risk factors for postoperative exacerbation of interstitial pneumonia in patients with interstitial lung disease must be identified. We analyzed 22 patients diagnosed as having lung cancer with interstitial lung disease who underwent surgical treatment at the Kitasato University Hospital. Among the patients with lung cancer and interstitial lung disease, 5 patients $(22.7 \%)$ had postoperative exacerbation of interstitial pneumonia. The prognosis of the patients with postoperative exacerbation was significantly poorer than that of patients without. Patients with postoperative exacerbation had a significantly higher age ( $\geq 75$ years) and a significantly lower frequency of postoperative administration of steroid than patients without postoperative exacerbation. Almost all patients with postoperative exacerbation underwent lobectomy, had elevated KL-6 levels in the serum pre-operatively, and had significantly advanced stages of disease. Of the 5 patients with postoperative exacerbation, 2 had a history of inflammation prior to their exacerbation: 1 had a common cold and the other pyothorax. In patients with lung cancer and interstitial lung disease, advanced age, advanced stage disease, no postoperative administration of steroid and a pre-operative episode of inflammation are all risk factors for postoperative exacerbation of interstitial pneumonia.
\end{abstract}

\section{Introduction}

Postoperative exacerbation of interstitial pneumonia in patients with lung cancer and interstitial lung disease has emerged as a serious problem. Postoperative interstitial pneumonia is a

Correspondence to: Dr Akira Iyoda, Department of Thoracic Surgery, Kitasato University, School of Medicine, 1-15-1, Kitasato, Minami-ku, Sagamihara, Kanagawa 252-0374, Japan

E-mail: aiyoda@med.kitasato-u.ac.jp

Key words: lung cancer, interstitial lung disease, postoperative exacerbation life-threatening complication with a high rate of mortality following surgery (1-3). Since it is difficult for patients with interstitial lung disease to undergo chemotherapy, radiation therapy or both, for their lung cancers, due to a high rate of exacerbation of interstitial pneumonia after treatment, surgical resection is sometimes the sole method of treatment for these patients. Moreover, performing surgical resection for patients with interstitial lung disease cannot be avoided when they have perquisite clinical stages and no other limitations. However, special care must be taken in the surgical management of patients with lung cancer and interstitial lung disease, as patients with interstitial lung disease have a high risk of postoperative exacerbation of interstitial pneumonia (4). Therefore, the risk factors for postoperative exacerbation of interstitial pneumonia in patients with interstitial lung disease must be identified. However, there are relatively few reports regarding the postoperative exacerbation of interstitial pneumonia. In this study, the predictive markers of postoperative exacerbation of interstitial pneumonia were investigated in patients with lung cancer and interstitial lung disease.

\section{Patients and methods}

We analyzed 22 patients diagnosed with lung cancer with interstitial lung disease who underwent surgical treatment at the Kitasato University Hospital between 1990 and 2009. Pre-operatively, interstitial lung diseases were detected by prior patient history, pre-operative chest X-ray or pre-operative computed tomography (CT) based on the ATS/ERS statement (5), and confirmed by pathological diagnoses from the resected specimens. Acute exacerbation of interstitial pneumonia was diagnosed when patients had intensified dyspnea, a decrease in arterial oxygen tension or an increased fine crackle on auscultation with an increased interstitial shadow on chest CT. Patients with collagen diseases were excluded from this study, as patients with these diseases may manifest different clinical features.

The following patient parameters were investigated from the medical records: gender, age, smoking history, clinical staging, pre-operative symptoms, site of tumors, pre-operative serum marker levels of Krebs von den Lungen-6 (KL-6), surgical procedure, pathological findings, including tumor size, time of death, latest follow-up day, postoperative interstitial pneumonia, administration of steroid and outcome. 
Table I. Patient characteristics $(n=22)$.

\begin{tabular}{lr}
\hline Factor & No. $(\%)$ \\
\hline Age & \\
Mean \pm SD & $69.9 \pm 6.9$ \\
Gender & \\
Male & $19(86.4)$ \\
Female & $3(13.6)$ \\
Site & \\
Right & $14(63.6)$ \\
Left & $8(36.4)$ \\
Clinical stage & \\
IA & $7(31.8)$ \\
IB & $12(54.5)$ \\
IIB & $2(9.0)$ \\
IIIA & $1(4.5)$ \\
Smoking & \\
Smoker & $16(72.7)$ \\
Ex-smoker & $6(27.3)$ \\
Surgery & \\
Lobectomy & $17(77.3)$ \\
Wedge resection & $5(22.7)$ \\
Postoperative exacerbation & \\
+ & $5(22.7)$ \\
- & $17(77.3)$ \\
\hline
\end{tabular}

Statistical analysis. Fisher's exact test was used to compare binomial proportions. The $\chi^{2}$ test was used to assess differences in treatment. A P-value of $<0.05$ was considered statistically significant.

\section{Results}

The characteristics of the patients are listed in Table I. All 22 patients had a history of smoking. Five patients $(22.7 \%)$ had postoperative exacerbation of interstitial pneumonia. There were no significant differences in gender, sites of lung cancers, pre-operative lung function, smoking history or the levels of lymph node dissection between the group with postoperative exacerbation of interstitial pneumonia and the group without. Among the patients with postoperative exacerbation, 4 patients were aged $\geq 75$ years; there was a significantly higher rate of patients $\geq 75$ years of age than among those without postoperative exacerbation. Patients with postoperative exacerbation presented with significantly advanced clinical stages compared to patients without postoperative exacerbation, and most patients with postoperative exacerbation underwent lobectomy. All patients with postoperative exacerbation had elevated serum KL-6 levels (Table II). Moreover, only 2 patients with postoperative exacerbation had prophylactic administration of steroid postoperatively, which was a significantly lower rate compared to patients without postoperative exacerbation. In 5 patients with postoperative exacerbation, 2 had a history of inflammation just prior to the exacerbation: 1 had a common cold and the other had pyothorax. The prognosis of patients

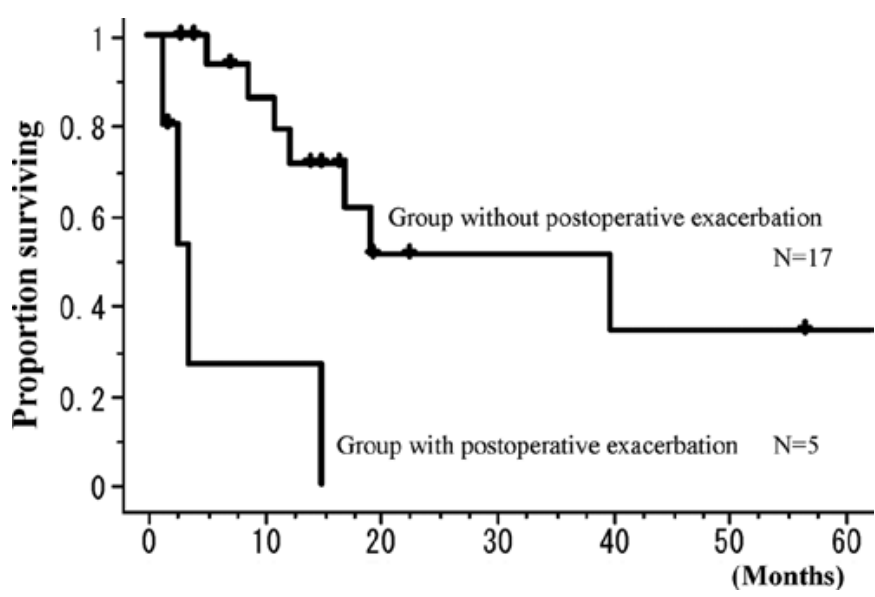

Figure 1. Overall survival curves for patients with postoperative acute exacerbation and patients without. Patients with postoperative exacerbation had a significantly poorer prognosis than patients without $(\mathrm{P}=0.0252)$.

with postoperative exacerbation was significantly poorer than that of patients without postoperative exacerbation $(\mathrm{P}=0.0252$, Fig. 1).

\section{Discussion}

At present, patients with various complications must undergo resection for lung cancer. Nakajima et al (6) reported that patients with lung cancer and collagen disease were not contraindicated to undergo surgical resection for lung carcinoma, although they frequently had interstitial pneumonia as pointed out in the resected specimens. Kutlu et al (1) investigated the frequency and mortality of acute lung injury and acute respiratory distress syndrome in patients after pulmonary resection, and reported that 45 (3.9\%) of 1,139 resected cases had postoperative acute lung injury or acute respiratory distress syndrome, while $29(64.4 \%)$ of these 45 patients died. Ruffini et al (2) reported that $27(2.2 \%)$ of 1,221 cases had postoperative acute lung injury or acute respiratory distress syndrome, and that $14(51.9 \%)$ of those 27 patients died. Muraoka et al (7) reported that $9(56.3 \%)$ of 16 patients died due to respiratory failure.

Therefore, postoperative exacerbation of interstitial pneumonia is a serious problem after pulmonary resection. However, little is known regarding postoperative exacerbation of interstitial pneumonia; in particular, the cause and the risk factors for interstitial pneumonia remain unknown (7). In various surgical approaches for lung cancers, Koizumi et al (8) compared the use of video-assisted thoracoscopic surgery to posterolateral thoracotomy or muscle-sparing thoracotomy and reported that the use of video-assisted thoracoscopic surgery did not prevent acute exacerbation of postoperative interstitial pneumonia. Therefore, postoperative acute interstitial pneumonia may be related to factors other than the surgical approach.

Takeda et al (3) reported that the measurement of serum KL-6 levels is useful to assess the activity of interstitial pneumonia. Moreover, Muraoka et al (7) reported that factors including a tumor located on the right side, pre-operative radiation or chemotherapy, pneumonectomy, blood transfusion and intraoperative complications were independent risk factors for 
Table II. Comparison between patients with and without postoperative exacerbation.

\begin{tabular}{|c|c|c|c|}
\hline Factor & $\begin{array}{l}\text { Postoperative exacerbation }(+) \\
\qquad(\mathrm{n}=5)\end{array}$ & $\begin{array}{l}\text { Postoperative exacerbation (-) } \\
\qquad(\mathrm{n}=17)\end{array}$ & P-value \\
\hline Age (years) & & & 0.0207 \\
\hline$\geq 75$ & 4 & 3 & \\
\hline$<75$ & 1 & 14 & \\
\hline Gender & & & $>0.9999$ \\
\hline Male & 4 & 15 & \\
\hline Female & 1 & 2 & \\
\hline Site & & & $>0.9999$ \\
\hline Right & 3 & 11 & \\
\hline Left & 2 & 6 & \\
\hline Clinical stage & & & 0.0344 \\
\hline IA & 2 & 5 & \\
\hline IB & 1 & 11 & \\
\hline IIB & 2 & 0 & \\
\hline IIIA & 0 & 1 & \\
\hline \multicolumn{4}{|l|}{ Lung function } \\
\hline $\mathrm{VC}(\mathrm{ml})$ & 2,753 & 3,081 & 0.3522 \\
\hline FEV1.0 (ml) & 2,158 & 2,306 & 0.5360 \\
\hline $\mathrm{PaO}_{2}$ (torr) & 77.1 & 81.6 & 0.4828 \\
\hline Smoking & & & $>0.9999$ \\
\hline Smoker & 4 & 12 & \\
\hline Ex-smoker & 1 & 5 & \\
\hline Surgery & & & $>0.9999$ \\
\hline Lobectomy & 4 & 13 & \\
\hline Wedge resection & 1 & 4 & \\
\hline Lymph node dissection & & & 0.6980 \\
\hline ND0 & 2 & 7 & \\
\hline ND1 & 3 & 8 & \\
\hline ND2 & 0 & 2 & \\
\hline KL-6 & & & 0.5165 \\
\hline High & 4 & 8 & \\
\hline Low & 0 & 4 & \\
\hline Postoperative steroid & & & 0.0239 \\
\hline Yes & 2 & 16 & \\
\hline No & 3 & 1 & \\
\hline
\end{tabular}

VC, vital capacity; FEV1.0, forced expiratory volume in one second; $\mathrm{PaO}_{2}$, arterial gas tension of $\mathrm{O}_{2}$.

the incidence of acute interstitial pneumonia. Koizumi et al (8) reported that exertion dyspnea (Hugh-Jones classification), serum C-reactive protein, serum lactate dehydrogenase and total lung capacity were considered to be pre-operative risk factors of acute exacerbation. Chida et al (9) reported that 11 of 15 patients with postoperative interstitial pneumonia were found to have interstitial pneumonia in pre-operative CT scans, and patients with findings of interstitial pneumonia in pre-operative CT scans had a significantly higher rate of postoperative acute respiratory distress syndrome (ARDS) than did patients with no such findings. They also reported that induction chemotherapy or chemoradiotherapy was a risk factor of postoperative ARDS (9).
In the present study, we selected patients with interstitial pneumonia pre-operatively and examined risk factors of postoperative exacerbation. The limitations of this study were that it was retrospective and that the number of patients was limited. However, the rate of postoperative exacerbation was high and, therefore, patients with pre-operative interstitial lung disease have a severe risk for postoperative exacerbation of interstitial pneumonia. In the present study, patients with postoperative exacerbation included patients with advanced age, those with a high rate of elevated serum KL-6, those who underwent lobectomy, and patients who did not receive steroid administration. Thus, special attention should be paid to postoperative exacerbation of interstitial pneumonia for patients 
with the above factors. Despite the limitation of the relatively small patient population, the occurrence of inflammation just prior to the postoperative exacerbation in the 2 patients with postoperative exacerbation was evaluated. Consequently the existence of inflammation may likely be one of the risk factors for postoperative exacerbation.

\section{Acknowledgements}

This study was supported in part by a Grant-in-aid for Scientific research (C) 21591822 of the Japanese Ministry of Education, Culture, Sports, Science, and Technology. The authors thank Mr. Robert E. Brandt, CEO, MedEd Japan, for the editorial assistance in the preparation of the manuscript.

\section{References}

1. Kutlu CA, Williams EA, Evans TW, Pastorino U and Goldstraw P: Acute lung injury and acute respiratory distress syndrome after pulmonary resection. Ann Thorac Surg 69: 376-380, 2000.

2. Ruffini E, Parola A, Papalia E, Filosso PL, Mancuso M, Oliaro A, Actis-Dato G and Maggi G: Frequency and mortality of acute lung injury and acute respiratory distress syndrome after pulmonary resection for bronchogenic carcinoma. Eur $\mathbf{J}$ Cardiothorac Surg 20: 30-36, 2001.

3. Takeda S, Maeda H, Sawabata N, Kitada S, Mori M, Takashima S and Matsubara Y: Clinical impact of interstitial pneumonia following surgery for lung cancer. Thorac Cardiovasc Surg 54: 268-272, 2006.
4. Chiyo M, Sekine Y, Iwata T, Tatsumi K, Yasufuku K, Iyoda A, Otsuji M, Yoshida S, Shibuya K, Iizasa T, Saitoh Y and Fujisawa T: Impact of interstitial lung disease on surgical morbidity and mortality for lung cancer: analyses of short-term and long-term outcomes. J Thorac Cardiovasc Surg 126: 1141-1146, 2003.

5. American Thoracic Society; European Respiratory Society: American Thoracic Society/European Respiratory Society International Multidisciplinary Consensus Classification of the Idiopathic Interstitial Pneumonias. This joint statement of the American Thoracic Society (ATS), and the European Respiratory Society (ERS) was adopted by the ATS board of directors, June 2001 and by the ERS Executive Committee, June 2001. Am J Respir Crit Care Med 165: 277-304, 2002

6. Nakajima J, Takamoto S, Murakawa T, Fukami T and Sano A: Is interstitial pneumonia in patients with collagen diseases a contraindication to lung cancer surgery? Surg Today 37: 14-18, 2007.

7. Muraoka M, Tagawa T, Akamine S, Oka T, Tsuchiya T, Araki M, Hayashi $\mathrm{T}$ and Nagayasu T: Acute interstitial pneumonia following surgery for primary lung cancer. Eur J Cardiothorac Surg 30: 657-662, 2006.

8. Koizumi K, Hirata T, Hirai K, Mikami I, Okada D, Yamagishi S, Kawashima T, Nakajima Y and Shimizu K: Surgical treatment of lung cancer combined with interstitial pneumonia: the effect of surgical approach on postoperative acute exacerbation. Ann Thorac Cardiovasc Surg 10: 340-346, 2004.

9. Chida M, Ono S, Hoshikawa Y and Kondo T: Subclinical idiopathic pulmonary fibrosis is also a risk factor of postoperative acute respiratory distress syndrome following thoracic surgery. Eur J Cardiothorac Surg 34: 878-881, 2008. 